\title{
Theoretical Challenges in neutrinoless double beta decay
}

\author{
Sabin Stoica* \\ Horia Hulubei Foundation, P.O. Box MG12 and \\ Horia Hulubei National Institute of Physics and Nuclear Engineering, P.O. Box MG6, \\ 077125 Bucharest-Magurele, Romania \\ E-mail: Sstoica53@gmail.com
}

\begin{abstract}
Neutrinoless double beta $(0 v \beta \beta)$ decay is a beyond Standard Model (BSM) process whose discovery would clarify if the lepton number is conserved, decide on the neutrinos character (are they Dirac or Majorana particles?) and give a hint on the scale of their absolute masses. Theoretically, the study of the $0 v \beta \beta$ decay involves the accurate computation of the nuclear matrix elements (NME) and phase space factors (PSF), two key quantities entering the lifetime formulas of this process. In this paper I make first a short review on the actual challenges to calculate the NME and PSF for double-beta decay (DBD). Then, I discuss the influence that different nuclear approximations and parameters have on the computed values of these quantities, outlining the advantage to calculate simultaneously products of NME and PSF, instead of providing separately their values. This would help at a better interpretation of the DBD experimental data.
\end{abstract}

The 26th International Nuclear Physics Conference

11-16 September, 2016

Adelaide, Australia

${ }^{*}$ Speaker. 


\section{Introduction}

Double beta decay (DBD) is a rare nuclear decay by which an even-even nucleus transforms into another even-even nucleus with the same mass $\mathrm{A}$ but with its nuclear charge $\mathrm{Z}$ changed by two units. One can classify the theoretical possible DBD modes according to the number and type of the leptons released in the decay. Thus, one can have the so-called two neutrino double beta decay $(2 v \beta \beta)$ modes, where in the final states electrons or positrons are emitted besides antineutrinos or neutrinos, and the $0 v \beta \beta$ decay modes, where only electrons/positrons are emitted, without anti-neutrinos/neutrinos in the final states. The $2 v \beta \beta$ are decay modes that conserve the lepton number and are allowed in the initial formulation of the Standard Model (SM). There are already measurements for eleven isotopes, with lifetime values ranging between $10^{18}-10^{24} \mathrm{yr}$. By contrary, the $0 v \beta \beta$ decay modes have not been discovered yet, but are very searched by many experiments. They are of great interest because can provide us with important information about the lepton number conservation and neutrino properties as, for example, the neutrinos character (are they Dirac or Majorana particles?) and their absolute mass [四]-[]. Theoretically, these decay modes can occur through several mechanisms, the most investigated being the exchange of light left-handed $(\mathrm{LH})$ neutrinos between two nucleons inside the nucleus. In this paper I refer to the $0 v \beta \beta$ decay mode with emission of two electrons that occur through this mechanism. The lifetime for this decay mode can be expressed as a product of three factors: a PSF, depending on the $Q_{\beta \beta}$ the energy of the decay and on the nuclear charge Z, a factor representing the NME and a Majorana neutrino mass parameter which is related to the elements of the neutrino mixing matrix and to the absolute neutrino mass eigenvalues [四]-[]. To derive accurate neutrino mass parameters and/or predict $0 v \beta \beta$ decay lifetimes, one needs precise calculations of both NME and PSF. The NME computation for DBD is a well-known theoretical challenge dating long time and that is not yet satisfactory resolved. There are several nuclear structure methods for these calculations, the most employed being proton-neutron Quasi Random Phase Approximation (pnQRPA) [[] $]$-[四],

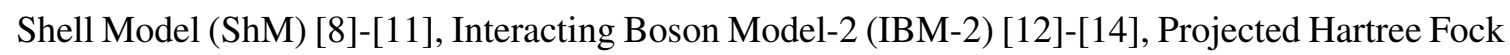
Bogoliubov (PHFB) [ए5] and Generating coordinate method (GCM) method [ए6]. At present there are still large differences in the literature between the NME values computed by different groups and with different methods, and these have been largely discussed in the literature (see, for example refs. [] and [1]7] and references there in). These differences come, on the one side, from the basic approximations specific to each method and, on the other side, from the different nuclear approximations and input parameters used in computation. In this paper I refer on the last ones. Concerning the PSF, they have been calculated since long time using different approximations [18]]-[23], and they were considered until recently to be calculated with enough precision. However, when they were recently recalculated with an improved approach, by using exact electron Dirac wave functions (w.f.), taking into account the finite nuclear size and electron screening effects [24], discrepancies were found in comparison with older calculations, especially for heavier nuclei. We also recalculated them by developing new routines for computing the relativistic (Dirac) electron w.f., with inclusion of the nuclear finite size and screening effects and, in addition, with the use of a Coulomb potential derived from a realistic proton density distribution in the daughter nucleus [25]-[26] and up-dated $Q_{\beta \beta}$-values, and we found similar discrepancies. Hence, we consider, it is worth to take into account these new calculations for extracting Majorana neutrino mass 
parameters.

In this paper I present recent calculations of the NME and PSF for $0 v \beta \beta$ decay, performed by our group, in the hypothesis that this decay mode occurs by exchange of light LH neutrinos between two nucleons inside the nucleus. The NME for three isotopes, i.e. ${ }^{48} \mathrm{Ca},{ }^{76} \mathrm{Ge}$ and ${ }^{82} \mathrm{Se}$, have been computed with a ShM code developed recently [27]-[28]. Also, I discuss the consensus on the use of different nuclear structure ingredients/parameters in the NME computation. Concerning the PSF, their values are computed with the code described in Refs. [25]-[26] but with an improved numerical precision. Then, I present new limits of the light Majorana neutrino mass parameter derived from the analysis of the $0 v \beta \beta$ decay of nine isotopes.

\section{Theoretical formalism}

The lifetimes for $0 v \beta \beta$ decay can be expressed as [四:

$$
\left(T_{1 / 2}^{0 v}\right)^{-1}=G^{0 v}\left(Q_{\beta \beta}, Z\right) g_{A}^{4}\left|M^{0 v}\right|^{2}\left(\left\langle m_{v}\right\rangle / m_{e}\right)^{2},
$$

where $G^{0 v}$, expressed in units of $[y r]^{-1}$, are the PSF for this decay mode, $m_{e}$ is the electron mass and $\left\langle m_{v}\right\rangle$ is the Majorana light neutrino mass parameter [四]. $M^{0 v}$ are the NME, which depend on the nuclear structure of the parent and daughter nuclei:

$$
M_{\alpha}^{0 v}=\sum_{m, n}\left\langle 0_{f}^{+}\left\|\tau_{-m} \tau_{-n} O_{m n}^{\alpha}\right\| 0_{i}^{+}\right\rangle
$$

where $\alpha=G T, F, T$ are the contributions associated with the Gamow-Teller $(G T)$, Fermi $(F)$ and Tensor $(T)$ two-body transition operators $O_{m n}^{\alpha}$, and the summation is performed over all the nucleon states. The computation of the reduced matrix elements of the operators $O^{\alpha}$ can be decomposed into products of reduced matrix elements within the spin and relative coordinate spaces. Their explicit expressions can be found, for example, in refs. [四, [प]]:

$$
O_{12}^{G T}=\sigma_{1} \cdot \sigma_{2} H(r), \quad O_{12}^{F}=H(r), \quad O_{12}^{T}=\sqrt{2 / 3}\left[\sigma_{1} \times \sigma_{2}\right]^{2} \cdot(r / R) H(r) C^{(2)}(\hat{r})
$$

The most difficult is the computation of the radial part of these two-body transition operators, which contains the neutrino potentials, defined by integrals over momentum carried by the virtual neutrino exchanged between the two nucleons [焑]:

$$
H_{\alpha}(r)=\frac{2 R}{\pi} \int_{0}^{\infty} j_{i}(q r) \frac{h_{\alpha}(q)}{\omega} \frac{1}{\omega+\langle E\rangle} q^{2} d q
$$

where $R=r_{0} A^{1 / 3} \mathrm{fm}\left(r_{0}=1.2 \mathrm{fm}\right), \omega=\sqrt{q^{2}+m_{v}^{2}}$ is the neutrino energy and $j_{i}(q r)$ is the spherical Bessel function ( $\mathrm{i}=0,0$ and 2 for GT, F, and T, respectively). Usually, in calculations one uses the closure approximation which consists in the replacement of the excitation energies of the states from the intermediate odd-odd nucleus contributing to the decay, by an average expression $\langle E\rangle$. The detailed expressions of $h_{\alpha}(\alpha=G T, F, T)$ can be found, for example, in refs. [四], [罒], [四]. They contain nuclear ingredients such as the finite nucleon size (FNS) and short range correlations (SRC) effects, as well as the inclusion of higher order terms in the nuclear currents (HOC), which are 
important for an accurate computation of the NME. The FNS effect is taken into account through nucleon form factors, $G_{V}$ and $G_{A}$, which depend on the momentum:

$$
G_{A}\left(q^{2}\right)=g_{A}\left(\Lambda_{A}^{2} /\left(\Lambda_{A}^{2}+q^{2}\right)\right)^{2}, G_{V}\left(q^{2}\right)=g_{V}\left(\Lambda_{V}^{2} /\left(\Lambda_{V}^{2}+q^{2}\right)\right)^{2}
$$

In calculations either the quenched $\left(g_{A}=1\right)$ or unquenched $\left(g_{A}=1.25-1.273\right)$, values of the axial-vector constant have been used, while the values of the cut-off parameters are usually taken as $\Lambda_{V}=850 \mathrm{MeV}$ and $\Lambda_{A}=1086 \mathrm{MeV}$ [四]. The SRC effects are included by correcting the single particle w. f.: $\psi_{n l}(r) \rightarrow[1+f(r)] \psi_{n l}(r)$. The correlation function $f(r)$ can be parametrized in different ways: the Jastrow prescription with the i) Miller-Spencer (MS) parametrization [29] and the CCM parameterizations, derived with realistic ii) CD-Bonn and iii) AV18 NN potentials [圆]:

$$
f(r)=-c \cdot e^{-a r^{2}}\left(1-b r^{2}\right)
$$

The three parameterizations mentioned above are associated with different sets of the a, b, c parameters. We mention that another method, the unitary correlation operator method (UCOM) is also successfully used to include SRC effects in nuclear structure calculations for DBD [四]. The inclusion of HOC brings additional terms in the $h_{G T}$ component and leads to the appearance of the $h_{T}$ component in the expressions of the neutrino potentials, as it is described in detail in refs. [四], [BO]]. Besides the nuclear effects mentioned above, a number of input parameters as $g_{A}, r_{0}$, $\left(\Lambda_{A}, \Lambda_{B}\right)$ and $<E>$ are involved in calculations, as well. The use of different values for these parameters may result in important differences in the calculated NME values. For example, the use of a quenched or an unquenched value for $g_{A}$ is still an unsolved matter.

\section{Results and discussions}

The largest uncertainties in the derivation of the Majorana neutrino mass parameter and/or prediction of $0 v \beta \beta$ decay half-lives come from the NME computation. Their values depend on the nuclear method and on the different nuclear ingredients/parameters used in calculations. Fortunately, at present there is a consensus on the use of the nuclear ingredients/parameters [B]] which helps to restrict the range of the different NME values from literature. For example, one recommends the inclusion in calculation of the HOC, FNS and the use of softer parameterizations like CCM [國] or UCOM [四] for SRC. Concerning the input nuclear parameters, one recommends the use of an unquenched value for the axial vector constant $\left(g_{A}=1.25-1.273\right)$ [[3] $)$, and a value of $r_{0}=1.2 \mathrm{fm}$ for the nuclear radius constant. Also, one can mention that the results are less sensitive to the changes of $\langle E\rangle$ value (within a few $\mathrm{MeV}$ ), used in the closure approximation, and to (small) variations of the values of cut-off parameters $\Lambda_{V, A}$. According to this consensus, we display in Table 1 the NME values obtained with different nuclear methods. The first row contains our results for ${ }^{48} \mathrm{Ca},{ }^{76} \mathrm{Ge}$ and ${ }^{82} \mathrm{Se}$, performed with the code described in more detail in [27]-[28], while the NME values for the other nuclei are taken from the references indicated in parenthesis. The NME values written in parenthesis for ${ }^{76} \mathrm{Ge}$ and ${ }^{82} \mathrm{Se}$ isotopes represent the values obtained with QRPA methods, when the s.p. energies were adjusted to the occupancy numbers reported in ref. [33]. One remarks, the NME values obtained with QRPA and ShM methods get closer when s.p. occupancies are adjusted to experiment. This is an important step in putting in agreement the 
Table 1: The NME obtained with different methods and with softer SRC parametrizations, specified in the second column. In calculations an unquenched value of $g_{A}$ was used.

\begin{tabular}{|c|c|c|c|c|c|c|c|c|c|c|}
\hline Method & SRC & ${ }^{48} \mathrm{Ca}$ & ${ }^{76} \mathrm{Ge}$ & ${ }^{82} \mathrm{Se}$ & ${ }^{96} \mathrm{Zr}$ & בM 100 & $116 r d$ & ${ }^{130} \mathrm{Te}$ & ${ }^{136} \mathrm{Xe}$ & ${ }^{150} \mathrm{Nd}$ \\
\hline [28]]ShM & CD-Bonn & 0.81 & 3.13 & 2.88 & & & & \multicolumn{3}{|c|}{2.21 [B4] } \\
\hline [ए0]ShM & UCOM & 0.85 & $2.81-3.52$ & 2.64 & & & & 2.65 & 2.19 & \\
\hline [ए4]]IBM-2 & CD-Bonn & 2.38 & 6.16 & 4.99 & 3.00 & 4.50 & 3.29 & 4.61 & 3.79 & 2.88 \\
\hline [ए7]QRPA & CD-Bonn & & $5.93(3.27) 5$ & $30(4.54)$ & 2.19 & 4.67 & 3.72 & 4.80 & 3.00 & $3.16[35]$ \\
\hline [36] QRPA & UCOM & & $5.36(4.11)$ & 3.72 & 3.12 & 3.93 & 4.79 & 4.22 & 2.80 & \\
\hline [ए6] EDF & CD-Bonn & 2.37 & 4.60 & 4.22 & 5.65 & 5.08 & 4.72 & 5.13 & 4.20 & 1.71 \\
\hline [15]]PHFB & CD-Bonn & & & & 2.98 & 6.07 & & 3.98 & & 2.68 \\
\hline
\end{tabular}

Table 2: Majorana neutrino mass parameters together with the other components of the $0 v \beta \beta$ decay halflives: $Q_{\beta \beta}$, the experimental half-life limits, the PSF and the NME.

\begin{tabular}{|c|c|c|c|c|c|}
\hline Nucleus & $Q_{\beta \beta}[\mathrm{MeV}]$ & $T_{\exp }^{0 v}[y r]$ & $G^{0 v}\left[y r^{-1}\right]$ & $M^{0 v}$ & $\left\langle m_{v}\right\rangle[e V]$ \\
\hline${ }^{48} \mathrm{Ca}$ & 4.272 & $>5.810^{22}[\mathrm{~B} 7]$ & $2.46 \mathrm{E}-14$ & $0.81-0.85$ & $<[10.18-10.69]$ \\
\hline${ }^{76} \mathrm{Ge}$ & 2.039 & $>2.110^{25}$ [4] ] & $2.37 \mathrm{E}-15$ & $2.81-6.16$ & $<[0.24-0.52]$ \\
\hline${ }^{82} \mathrm{Se}$ & 2.995 & $>3.610^{23}[\mathrm{~B} 8]$ & $1.01 \mathrm{E}-14$ & $2.64-4.99$ & $<[1.09-2.05]$ \\
\hline${ }^{96} \mathrm{Zr}$ & 3.350 & $>9.210^{21}[\mathrm{BG}]$ & $2.05 \mathrm{E}-14$ & $2.19-5.65$ & $<[4.21-10.9]$ \\
\hline${ }^{100} \mathrm{Mo}$ & 3.034 & $>1.110^{24}[\mathrm{BB}]$ & $1.57 \mathrm{E}-14$ & $3.93-6.07$ & $<[0.41-0.63]$ \\
\hline${ }^{116} \mathrm{Cd}$ & 2.814 & $>1.710^{23}$ [44] & $1.66 \mathrm{E}-14$ & $3.29-4.79$ & $<[1.28-1.87]$ \\
\hline${ }^{130} \mathrm{Te}$ & 2.527 & $>2.810^{24}$ [43] & $1.41 \mathrm{E}-14$ & $2.65-5.13$ & $<[0.32-0.62]$ \\
\hline${ }^{136} \mathrm{Xe}$ & 2.458 & $>1.610^{25}[42]$ & $1.45 \mathrm{E}-14$ & $2.19-4.20$ & $<[0.16-0.31]$ \\
\hline${ }^{150} \mathrm{Nd}$ & 3.371 & $>1.810^{22}$ [40] & $6.19 \mathrm{E}-14$ & $1.71-3.16$ & $<[2.68-5.73]$ \\
\hline
\end{tabular}

ShM and QRPA results and should be verified for other isotopes as well. In Table 2 we present the results for the Majorana neutrino mass parameters $\left(\left\langle m_{v}\right\rangle\right)$, derived from Eq. (1) together with the values of $Q_{\beta \beta}$, PSF $\left(G^{0 v}\right)$, NME $\left(M^{0 v}\right)$, and the experimental half-lives $\left(T_{1 / 2}^{0 v}\right)$ for nine isotopes, for which data exist. The ranges of the NME values were taken from Table 1. According to the consensus concerning the use of different nuclear ingredients/parameters, we reduced the interval of their spread to about a factor of 2 (with one exception). This results in reducing the uncertainty in deriving the constraints on the light Majorana neutrino mass parameters, while taking into account NME values obtained with all the main nuclear methods from the market. The PSF values were obtained by recalculating them with our code, described in [25], but with an improved numerical precision obtained by enhancing the number of the interpolation points for each isotope, on a case-to-case basis, until the results become stationary. We remark that the obtained values are close to those reported previously in refs. [24] and [25], for the most of isotopes, but differ from other previous calculations, from Refs. [R2], [2]], [23], by up to $28 \%$. Hence, it is justified the PSF re-computation with improved methods and use of the new values in the derivation of upper limits for the light neutrino mass parameter. For the experimental half-lives we took the most recent results reported in literature. One observes that the stringent constraints are obtained from the ${ }^{136} \mathrm{Xe}$ isotope, followed by the ${ }^{76} \mathrm{Ge}$ one. This is due to both the experimental sensitivity of the DBD experiments that measure these isotopes and to the accuracy of the theoretical calculations of 
the corresponding PSF and NME quantities. It is worth to note that the experiments that measure these isotopes are already exploring the quasi-degenerate scenarios for the neutrino mass hierarchy (which is around $0.5 \mathrm{eV}$ ). We note, however, that for the prediction of the neutrino mass parameter it is rather important to have computed accurately and consistently the product of the NME and PSF, i.e. their values âĂŃâĂŃhave to be calculated with the same nuclear approximations and with the same values of the nuclear parameters mentioned above.

\section{Conclusions}

I reviewed the theoretical challenges in the study of the neutrinoless DBD, i.e. the computation of the NME and PSF for the $0 v \beta \beta$ decay mode, in the hypothesis that it occurs by exchange of light LH Majorana neutrinos between two nucleons inside the nucleus. The NME values for three isotopes, ${ }^{48} \mathrm{Ca},{ }^{76} \mathrm{Ge}$ and ${ }^{82} \mathrm{Se}$, were calculated with our code described in detail in [27][28]. The PSF were recalculated with an approach described in ref. [25] but with an improved numerical accuracy, with an increased number of interpolation points for each isotope. We used exact electron w.f. obtained by solving a Dirac equation when FNS and screening effects are included and, in addition, we used a Coulomb potential derived from a realistic proton distribution in the daughter nucleus was employed. Then, we derived new upper limits of the light Majorana neutrino parameter from the analysis of the $0 v \beta \beta$ decay of nine isotopes. For the other six isotopes the NME values were selected from literature taking advance of the existent consensus on the use of the nuclear ingredients, as HOC, FHS and SRC effects, and on the values of several nuclear input parameters, which are involved in calculations. This allows us to restrict the range of spread of the NME values, and thus to reduce the uncertainty in deriving constraints on the Majorana neutrino mass parameter. Our results may be useful to have an up-to-date image on the current neutrino mass sensitivity associated with $0 v \beta \beta$ decay measurements for different isotopes, and to better estimate the range of the neutrino masses that can be explored in the future DBD experiments.

\section{Acknowledgments}

This work was done with the support of the MEN and UEFISCDI through the project IDEIPCE-3-1318, contract Nr. 58/28.10/2011 and Project PN-09-37-01-02/2009.

\section{References}

[1] Vergados, J., Ejiri, H. and Simkovic, F., 2012, Rep. Prog. Phys. 75, 106301.

[2] Vogel, P., J.Phys. G: Nucl. Part. Phys. 39, 124002 (2012).

[3] Rodin, V.A., Faessler, A., Simkovic F. and Vogel, P. 2003, Phys. Rev. C 68, 044302; Nucl. Phys. A 793.

[4] Kortelainen, M., Civitarese, O., Suhonen, J. and Toivanen, J. 2007, Phys. Lett. B 647, 128; Kortelainen, M. and Suhonen, J. 2007, Phys. Rev. C 75, 051303; Phys. Rev. C 76, 024315.

[5] Simkovic, F., Faessler, A., Rodin, V.A., Vogel P. and Engel J. 2008, Phys. Rev. C 77, 045503.

[6] Simkovic, F., Faessler, A., Muther, H., Rodin, V.A. and Stauf M. 2009, Phys. Rev. C 79, 055501. 
[7] Stoica, S. and Klapdor-Kleingrothaus, H.V. 2001, Nucl. Phys. A 694, 269.

[8] Caurier, E., Zuker, A.P., Poves, A. and Martinez-Pinedo, G. 1994, Phys. Rev. C 50, 225; Retamosa, J., Caurier E. and Nowacki, F. 1995 it Phys. Rev. C 51, 371.

[9] Caurier, E., Menendez, J., Nowacki, F. and Poves, A. 2008, Phys. Rev. Lett. 100, 052503.

[10] Menendez, J., Poves, A., Caurier, E., and Nowacki, F. 2009, Nucl. Phys. A 818139.

[11] Horoi, M. and Stoica, S. 2010, Phys. Rev. C 81, 024321.

[12] Barea, J and Iachello, F. 2009, Phys. Rev. C 79, 044301.

[13] Barea, J., Kotila, J. and Iachello, F. 2012, Phys. Rev. Lett. 109, 042501.

[14] Barea, J., Kotila, J. and Iachello, F. 2013, Phys. Rev. C 87, 014315.

[15] Rath, P.K., Chandra, R., Chaturvedi, K., Lohani, P., Raina, P.K. and Hirsch, J.G. 2013, Phys. Rev. C 88, 064322 .

[16] Rodriguez, T.R. and Martinez-Pinedo, G. 2010, Phys. Rev. Lett. 105, 252503.

[17] Faessler, A., Rodin, V. and Simkovic, F. 2012, J. Phys. G 39, 124006.

[18] Primakov, H. and Rosen, S.P. 1959, Rep. Prog. Phys. 22, 121.

[19] Haxton, W.C. and Stephenson Jr., G.J. 1984, Prog. Theor. Part. Nucl. Phys. 12, 409

[20] Doi, M., Kotani, T. and Takasugi, E. 1985, Prog. Theor. Phys. Suppl. 83, 1.

[21] Doi, M. and Kotani, T. 1992, Prog. Theor. Phys. 87, 1207; ibidem 1993 89, 139.

[22] Tomoda, T. 1991, Rep. Prog. Phys. 54, 53.

[23] Suhonen, J. and Civitarese, O. 1998 Phys. Rep. 300, 123.

[24] Kotila, J. and Iachello, F. 2012, Phys. Rev. C 85, 034316.

[25] Stoica, S. and Mirea, M, 2013, Phys. Rev. C 88, 037303.

[26] Pahomi, T.E., Neacsu, A., Mirea, M. and Stoica, S. 2014, Rom. Rep. Phys. 66, 370.

[27] Neacsu, A., Stoica, S. and Horoi, M 2012, Phys. Rev. C 86, 067304.

[28] Neacsu, A. and Stoica, S. 2014, J. Phys. G: Nucl. Part. Phys. 41, 015201.

[29] Miller G. A. and Spencer J. E. 1976, Ann. Phys., NY 100, 562.

[30] Simkovic, F., Pantis G., Vergados J.D. and Faessler A, 1999, Phys. Rev. C 60, 055502.

[31] Giuliani, A. and Poves, A. 2012 AHEP 2012, 857016.

[32] Liu, J. et al. (UCNA Collaboration) 2010, Phys. Rev. Lett. 105, 181803.

[33] Schiffer, J.P. et al. 2008, Phys. Rev. Lett. 100, 112501; B.P. Kay et al. 2009, Phys. Rev. C 79, 021301(R).

[34] Horoi, M and Brown, B.A. 2013, Phys. Rev. Lett. 110, 222502.

[35] Fang, D.L., Faessler, A. and Rodin, V.A. 2010, Phys. Rev. C 82, 051301(R)

[36] Civitarese, O. and Suhonen, J. 2009 J. Phys: Conf. Series 173, 012012.

[37] Umehara, S. et al. 2008, Phys Rev C 78, 058501.

[38] Barabash, A.S. and Brudanin V. B. 2011, Phys. At. Nucl. 74, 312. 
[39] Argyriades, J. et al. (NEMO3 Collaboration) 2010, Nucl. Phys. A 847, 168.

[40] Argyriades et al. (NEMO3 Collaboration) 2009, Phys. Rev. C 80, 032501.

[41] Macolino, C. et al. (GERDA Collaboration) 2014, Mod. Phys. Lett. A 29, 1430001.

[42] Gando, A. et al. 2013, Phys. Rev. Lett. 110, 062502.

[43] Andreotti, E. 2011, Astropart. Phys. 34, 822.

[44] Danevich, F.A. et al. 2003, Phys. Rev. C 68, 035501. 